\title{
Predictors of pneumothorax following endoscopic valve therapy in patients with severe emphysema
}

\author{
This article was published in the following Dove Press journal: \\ International Journal of COPD \\ I August 2016 \\ Number of times this article has been viewed
}

\section{Daniela Gompelmann ${ }^{1,2, *}$ \\ Hyun-ju Lim ${ }^{3,4, *}$ \\ Ralf Eberhardt ${ }^{1,2}$ \\ Vasiliki Gerovasili ${ }^{5}$ \\ Felix JF Herth ${ }^{1,2}$ \\ Claus Peter Heussel ${ }^{2-4}$ \\ Monika Eichinger ${ }^{3,4}$}

'Pneumology and Critical Care Medicine, Thoraxklinik at University of Heidelberg, ${ }^{2}$ Translational Lung

Research Center Heidelberg, Member of the German Center for Lung Research, ${ }^{3}$ Diagnostic and Interventional Radiology with Nuclear Medicine, Thoraxklinik at University of Heidelberg, ${ }^{4}$ Diagnostic and Interventional Radiology, University of Heidelberg, Heidelberg, Germany; ${ }^{5}$ First Critical Care Department, National and Kapodistrian University of Athens, Athens, Greece

*These authors contributed equally to this work

Correspondence: Daniela Gompelmann Pneumology and Critical Care Medicine, Thoraxklinik at University of Heidelberg, Röntgenstr I, 69126 Heidelberg, Germany $\mathrm{Tel}+4962213968087$ Fax +49 622। 396 I 202 Email daniela.gompelmann@med. uni-heidelberg.de
Background: Endoscopic valve implantation is an effective treatment for patients with advanced emphysema. Despite the minimally invasive procedure, valve placement is associated with risks, the most common of which is pneumothorax. This study was designed to identify predictors of pneumothorax following endoscopic valve implantation.

Methods: Preinterventional clinical measures (vital capacity, forced expiratory volume in 1 second, residual volume, total lung capacity, 6-minute walk test), qualitative computed tomography (CT) parameters (fissure integrity, blebs/bulla, subpleural nodules, pleural adhesions, partial atelectasis, fibrotic bands, emphysema type) and quantitative CT parameters (volume and low attenuation volume of the target lobe and the ipsilateral untreated lobe, target air trapping, ipsilateral lobe volume/hemithorax volume, collapsibility of the target lobe and the ipsilateral untreated lobe) were retrospectively evaluated in patients who underwent endoscopic valve placement $(n=129)$. Regression analysis was performed to compare those who developed pneumothorax following valve therapy $(n=46)$ with those who developed target lobe volume reduction without pneumothorax $(\mathrm{n}=83)$.

Finding: Low attenuation volume $\%$ of ipsilateral untreated lobe (odds ratio $[\mathrm{OR}]=1.08$, $P=0.001)$, ipsilateral untreated lobe volume/hemithorax volume ( $\mathrm{OR}=0.93, P=0.017)$, emphysema type $(\mathrm{OR}=0.26, P=0.018)$, pleural adhesions $(\mathrm{OR}=0.33, P=0.012)$ and residual volume (OR $=1.58, P=0.012$ ) were found to be significant predictors of pneumothorax. Fissure integrity $(\mathrm{OR}=1.16, P=0.075)$ and 6 -minute walk test $(\mathrm{OR}=1.05, P=0.077)$ were also indicative of pneumothorax. The model including the aforementioned parameters predicted whether a patient would experience a pneumothorax $84 \%$ of the time (area under the curve $=0.84$ ).

Interpretation: Clinical and CT parameters provide a promising tool to effectively identify patients at high risk of pneumothorax following endoscopic valve therapy.

Keywords: endoscopic lung volume reduction, COPD, emphysema, pneumothorax, valve therapy

\section{Introduction}

Endoscopic valve therapy has evolved as a new therapeutic modality in patients with advanced chronic obstructive pulmonary disease (COPD) and emphysema. This technique involves the implantation of one-way valves in the emphysematous lung lobe. By allowing the air to exit during expiration, the valves lead to target lobe volume reduction (TLVR), whereby a complete lobar atelectasis represents the optimal result.

The first randomized controlled trials (RCTs), known as VENT ("Endobronchial Valves for Emphysema Palliation Trial") and Euro-VENT, demonstrated encouraging results concerning the safety and effectiveness of this procedure. ${ }^{1,2}$ Adverse events, including COPD exacerbations, pneumonia, mild hemoptysis, hypercapnia, and pneumothorax, were observed, but the rate of serious complications did not differ between the treatment and control groups. In these RCTs, 
the pneumothorax rate was $4.2 \%$ and $4.5 \%$ at 90 days following the intervention.

In VENT and Euro-VENT, complete interlobar fissures in the preinterventional multidetector computed tomography (MDCT), which is a surrogate for low interlobar collateral ventilation, and lobar occlusion were found to be predictors of a meaningful clinical outcome following valve placement. Therefore, only a complete occlusion of the target lobe in patients with low collateral ventilation was recommended; this was reevaluated in two recently published RCTs, the BeLieVeR-HIFI study and STELVIO., These trials confirmed the efficacy of valve therapy but revealed an increased pneumothorax rate of $8 \%-18 \%$. It is likely that a parenchymal rupture of the ipsilateral untreated lobe due to rapid expansion in the case of volume reduction of the treated lobe is the reason for the pneumothorax. In further trials, the authors reported even higher pneumothorax rates of $25 \%, 23 \%$, and $20 \% .{ }^{5-7}$ Thus, the optimized patient selection is not only associated with improved outcomes following valve placement but also implies a higher risk of pneumothorax. Completeness of the fissure is particularly considered to support optimized outcomes as well as the advent of pneumothorax.

As pneumothorax seems to occur only in patients with significant volume shift, it is assumed that they may nevertheless experience good clinical outcomes in case of persistent TLVR after recovering from pneumothorax. ${ }^{8}$ However, pneumothorax presents a severe complication that frequently requires chest tube insertion and is associated with immobilization, prolonged hospitalization, and further endoscopic or surgical interventions. ${ }^{9,10}$ Furthermore, tension pneumothorax may also present a life-threatening complication that may lead to a shift of the mediastinum and obstruction of venous return to the heart, compromising cardiovascular circumstances. Therefore, assessing the predictors of pneumothorax other than fissure integrity, which also predicts TLVR, is of great importance.

\section{Methods}

In this retrospective analysis, clinical and MDCT scan data of patients who underwent valve therapy were examined to determine predictors of pneumothorax following valve therapy. The protocol of this retrospective analysis was approved by the local Ethics Committee of Heidelberg (S-609/2012). The majority of the patients were treated within different prospective trials after written informed consent. As the data in this current analysis were retrospectively analyzed no further patient consent was required.

\section{Subjects and clinical parameters}

In this retrospective analysis, baseline clinical measures and MDCT scan data of 129 consecutive patients (mean age: 64 years, range $43-81$ years, sex: 50\% male) who experienced TLVR ( $n=83)$ or pneumothorax $(n=46)$ following valve therapy between 2009 and 2013 were assessed. The mean forced expiratory volume in 1 second $\left(\mathrm{FEV}_{1}\right)$ was $0.8 \pm 0.2$ $1(32 \% \pm 8 \%$ predicted) and the mean residual volume (RV) was $5.6 \pm 1.41(257 \% \pm 59 \%$ predicted $)$. All the patients experienced a complete occlusion of the target lobe by endobronchial (Pulmonx, Inc., Neuchatel, Switzerland) or intrabronchial valves (Olympus Corporation, Tokyo, Japan) at the Thoraxklinik at the University of Heidelberg. Prior to valve therapy, MDCT, including software analysis of the emphysema (YACTA, "yet another CT analyser"), and $99 \mathrm{~m}$-Technetium perfusion scan were performed to identify the target lobe. Sixty-one patients (47\%) experienced complete occlusion of the left lower lobe, 34 (26\%) of the left upper lobe, $20(16 \%)$ of the right upper lobe, $13(10 \%)$ of the right lower lobe, and one (1\%) of the right upper and middle lobes. TLVR was defined as lung parenchymal collapse with the development of a soft-tissue dense atelectasis at a lobar or segmental level on a follow-up chest $\mathrm{X}$-ray or CT scan.

Clinical parameters prior to valve therapy and 3 months following pneumothorax, including vital capacity (VC), forced expiratory volume in 1 second $\left(\mathrm{FEV}_{1}\right)$, residual volume (RV), total lung capacity (TLC), and the 6-minute walk test (6-MWT), were extracted from the patients' records. Descriptive parameters (target lobe) were also evaluated.

\section{MDCT parameters}

The qualitative and quantitative MDCT parameters were assessed on preinterventional baseline MDCT scans (64-slice Somatom Definition AS64, Siemens Medical Solution, Forchheim, Germany); the CT protocol varied with reconstruction slice thickness of 1.0 (filter I40f), and doses of $100 \mathrm{kV}, 117 \mathrm{mAs}_{\text {effective }}$ or $120 \mathrm{kV}, 70 \mathrm{mAs}_{\text {effective }}$. The qualitative MDCT parameters were visually assessed by one experienced chest radiologist. The quantitative CT measurements were obtained by using syngo.via software (Siemens Medical Solution), which provides automated three-dimensional quantifications for the assessment of emphysema. The mean lung density of the lobes, lobar volumes, low attenuation volumes (LAVs) and the lobar collapsibility were obtained. The LAV (lung volumes with attenuation values $<950$ Hounsfield units) and LAV\% (the ratio of LAV to the volume of the region of interest) that 
correlate with lung function parameters, visual CT emphysema scores, and the COPD assessment test were used to describe the extent and severity of emphysema. ${ }^{11-14}$

\section{Statistical analysis}

Univariate logistic regression models were fitted to assess the association between each of the 41 variables and pneumothorax following valve therapy. We also assessed whether each continuous variable had a linear or quadratic association with pneumothorax in the logit scale. Stepwise forward regression model was fitted to assess the association between possible predictor variables and pneumothorax. Variables with a $P$-value $<0.5$ were included in the stepwise forward regression model in the most appropriate form (linear or quadratic).
The paired Student's $t$-test was used to compare within-group differences in qualitative parameters. $P$-values $<0.05$ were considered to be significant. Receiver operating characteristic curves were created using the final model of predictors.

\section{Results}

The patient characteristics of the 83 subjects with a radiologically confirmed TLVR (Figure 1) and those of 46 patients who developed pneumothorax (Figure 2) are presented in Table 1 . On the baseline MDCT scans, fissure integrity varied from $80 \%$ to $100 \%$. The fissure integrity was between $90 \%$ and $100 \%$ in 121 patients and between $80 \%$ and $90 \%$ in eight patients. Overall, the median of fissure completeness was $100 \%$ (interquartile range 98.3 to 100 ).

Table I Patient characteristics

\begin{tabular}{|c|c|c|}
\hline \multirow[t]{2}{*}{ Baseline clinical measures and CT parameters } & \multirow{2}{*}{$\begin{array}{l}\text { Patients with } \\
\text { TLVR }(n=83) \\
\text { Mean } \pm \text { SD }\end{array}$} & \multirow{2}{*}{$\begin{array}{l}\text { Patients with } \\
\text { pneumothorax }(n=46) \\
\text { Mean } \pm \text { SD }\end{array}$} \\
\hline & & \\
\hline \multicolumn{3}{|l|}{ Clinical variables } \\
\hline $\mathrm{VC}(\mathrm{L})$ & $2.4 \pm 0.79$ & $2.5 \pm 0.78$ \\
\hline VC (\%) & $72.0 \pm 17.2$ & $72.7 \pm 18.5$ \\
\hline $\mathrm{FEV}_{1}(\mathrm{~L})$ & $0.8 \pm 0.24$ & $0.8 \pm 0.24$ \\
\hline $\mathrm{FEV}_{1}(\%)$ & $31.7 \pm 6.6$ & $31.1 \pm 9.2$ \\
\hline $\mathrm{RV}(\mathrm{L})$ & $5.4 \pm 1.30$ & $5.9 \pm 1.43$ \\
\hline RV (\%) & $246.9 \pm 42.5$ & $273.7 \pm 79.2$ \\
\hline TLC (L) & $7.8 \pm 1.71$ & $8.4 \pm 1.61$ \\
\hline TLC (\%) & $136.1 \pm 15.6$ & $145.2 \pm 26.9$ \\
\hline 6-MWT (m) & $28 I .1 \pm 107.4$ & $305.3 \pm 93.4$ \\
\hline \multicolumn{3}{|l|}{ Quantitative CT variables } \\
\hline MLD of the target lobe $(\mathrm{HU})$ & $-916 \pm 18$ & $-918 \pm 20$ \\
\hline MLD of the untreated ipsilateral lobe $(\mathrm{HU})$ & $-886 \pm 22$ & $-893 \pm 23$ \\
\hline Target lobe volume $(\mathrm{mL})$ & $\mathrm{I}, 70 \mathrm{I} \pm 466$ & $|, 83| \pm 5 \mid 5$ \\
\hline Volume of the untreated ipsilateral lobe $(\mathrm{mL})$ & $\mathrm{I}, 629 \pm 494$ & $\mathrm{I}, 6 \mathrm{I} 7 \pm 456$ \\
\hline LAV of the target lobe/target lobe volume (= LAV\% of the target lobe) (\%) & $51.2 \pm 13.6$ & $51.5 \pm 15.1$ \\
\hline $\begin{array}{l}\text { LAV of the untreated ipsilateral lobe/volume of the untreated ipsilateral } \\
\text { lobe (= LAV\% of the untreated ipsilateral lobe) }(\%)\end{array}$ & $31.3 \pm 11.6$ & $37.0 \pm 12.3$ \\
\hline Volume of the untreated ipsilateral lobe to the volume of the hemithorax & $48.6 \pm 8.4$ & $47.1 \pm 8.8$ \\
\hline Collapsibility of the target lobe & $195.5 \pm 152.1$ & $229.4 \pm 195.3$ \\
\hline Collapsibility of the untreated target lobe & $276.0 \pm 208.6$ & $281.0 \pm 137.8$ \\
\hline \multicolumn{3}{|l|}{ Qualitative CT parameters } \\
\hline Fissure integrity $(\%)$ & $97.4 \pm 5.1$ & $98.9 \pm 2.0$ \\
\hline Blebs/bulla - no of subjects (\%) & $7(8.4)$ & $3(6.5)$ \\
\hline Subpleural nodules - no of subjects (\%) & $12(14.5)$ & $6(13.0)$ \\
\hline Pleural adhesions - no of subjects (\%) & $53(63.9)$ & $19(41.3)$ \\
\hline Partial atelectasis - no of subjects (\%) & $8(9.6)$ & $7(15.2)$ \\
\hline Fibrotic band - no of subjects (\%) & $29(34.9)$ & $15(32.6)$ \\
\hline Bronchiectasis - no of subjects (\%) & $8(9.6)$ & $4(8.7)$ \\
\hline Rib fracture - no of subjects (\%) & $14(16.9)$ & $3(6.5)$ \\
\hline Panlobular type of emphysema - no of subjects (\%) & $49(59)$ & $22(47.8)$ \\
\hline
\end{tabular}

Note: Plus-minus values are mean \pm SD.

Abbreviations: 6-MWT, 6-minute walk test; CT, computed tomography; FEV , forced expiratory volume in I second; HU, Hounsfield unit; LAV, low attenuation volume; MLD, mean lung density; RV, residual volume; SD, standard deviation; TLC, total lung capacity; TLVR, target lobe volume reduction; VC, vital capacity. 


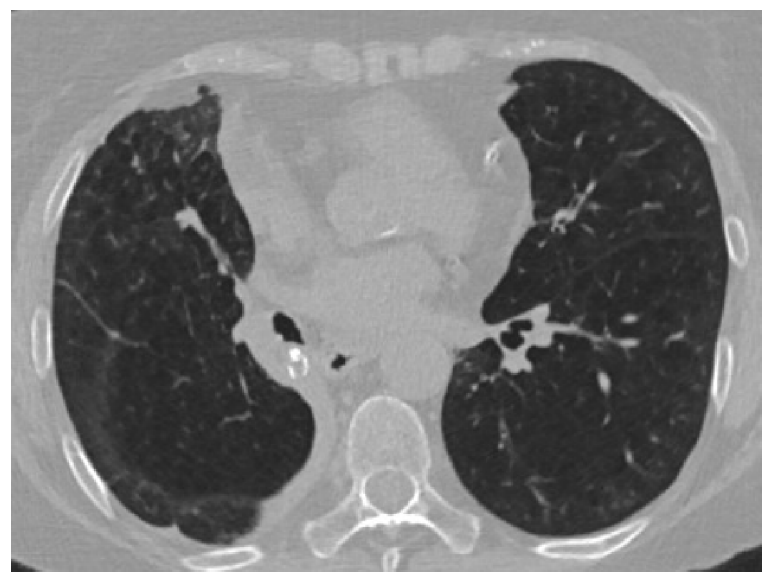

Figure I Multidetector computed tomography image. Note: Lobar atelectasis of the right lower lobe following valve implantation.

Thirty-eight of the 46 patients $(83 \%)$ who developed pneumothorax underwent chest tube insertion. In 18 patients (39\%), valve explantation was necessary. Eight patients (17\%) underwent additional video-assisted thoracoscopy to seal the fistula. Three months after recovering from pneumothorax, patients experienced slight, but not clinically relevant improvements in lung function parameters, exercise capacity and dyspnoe score (Table 2).

After the multivariable regression analysis (Table 3), the LAV\% of the untreated ipsilateral lobe, the volume of the untreated ipsilateral lobe to volume of the hemithorax, the predominant type of emphysema, the presence of pleural adhesions, and the residual volume were variables that were independently associated with pneumothorax, adjusting for the target lobe; the 6-MWT and the fissure integrity were borderline non-significant. Thereby, residual volume (odds ratio

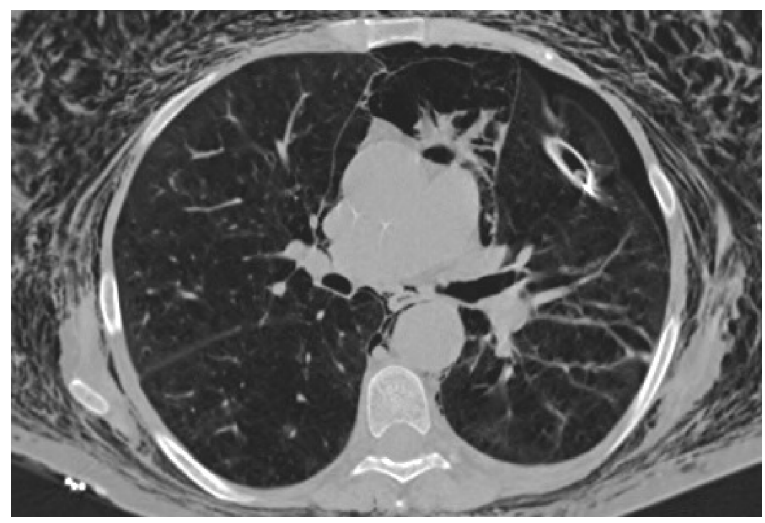

Figure 2 Multidetector computed tomography image.

Notes: Pneumothorax with subsequent severe bilateral subcutaneous emphysema 3 days following valve placement in the left lower lobe. The lung is reinflated after chest tube insertion.
Table 2 Clinical outcome of patients who developed pneumothorax

\begin{tabular}{|c|c|c|c|c|}
\hline $\begin{array}{l}\text { Clinical } \\
\text { parameter }\end{array}$ & $\mathbf{n}$ & $\begin{array}{l}\text { Baseline } \\
\text { mean } \pm \text { SD }\end{array}$ & $\begin{array}{l}\text { Three months following } \\
\text { pneumothorax } \\
\text { mean } \pm \text { SD }\end{array}$ & $P$-value \\
\hline VC (L) & 36 & $2.54 \pm 0.79$ & $2.52 \pm 0.72$ & 0.856 \\
\hline VC (\%) & 35 & $72 \pm 19$ & $72 \pm 18$ & 0.855 \\
\hline $\mathrm{FEV}_{1}$ (L) & 36 & $0.8 I \pm 0.24$ & $0.83 \pm 0.29$ & 0.286 \\
\hline $\mathrm{FEV}_{1}(\%)$ & 36 & $30 \pm 9$ & $31 \pm 10$ & 0.270 \\
\hline $\mathrm{RV}(\mathrm{L})$ & 34 & $5.97 \pm 1.33$ & $5.65 \pm 1.50$ & 0.060 \\
\hline RV (\%) & 35 & $275 \pm 60$ & $257 \pm 60$ & 0.029 \\
\hline TLC (L) & 34 & $8.57 \pm 1.60$ & $8.25 \pm 1.76$ & 0.040 \\
\hline TLC (\%) & 35 & $144 \pm 22$ & $138 \pm 17$ & 0.024 \\
\hline 6-MWT (m) & 31 & $320 \pm 90$ & $330 \pm 87$ & 0.548 \\
\hline mMRC (pts) & 26 & $2.25 \pm 1.09$ & $1.81 \pm 1.16$ & 0.442 \\
\hline
\end{tabular}

Note: Data were assessed 3 months following recovery from pneumothorax. Abbreviations: 6-MWT, 6-minute walk test; $\mathrm{FEV}_{1}$, forced expiratory volume in I second; RV, residual volume; SD, standard deviation; TLC, total lung capacity; VC, vital capacity; mMRC, modified Medical Research Council.

$[\mathrm{OR}]=1.58, P=0.012$ ) and untreated ipsilateral lobe $\mathrm{LAV} \%$ $(\mathrm{OR}=1.08, P=0.001)$ were associated with an increased risk of pneumothorax. In contrast, the presence of pleural adhesions (OR $=0.33, P=0.012)$, the presence of panlobular emphysema (OR $=0.26, P=0.018)$, and the volume of the untreated ipsilateral lobe to the volume of the hemithorax $(\mathrm{OR}=0.93$, $P=0.017)$ were associated with a decreased risk of pneumothorax. Finally, the 6-MWT $(\mathrm{OR}=1.05, P=0.077)$ and the fissure integrity $(\mathrm{OR}=1.16, P=0.075)$ tended to be associated with an increased risk of pneumothorax. The area under the curve using the full model with eight variables was 0.84 . This indicates that it is possible to predict whether a patient would experience a pneumothorax $84 \%$ of the time (Figure 3 ).

Table 3 Predictors for pneumothorax following valve therapy

\begin{tabular}{|c|c|c|c|}
\hline Pneumothorax & $\begin{array}{l}\text { Odds } \\
\text { ratio }\end{array}$ & $\begin{array}{l}95 \% \\
\text { confidence } \\
\text { interval }\end{array}$ & $P$-value \\
\hline Ipsilateral lobe LAV\% & 1.08 & $1.04-1.14$ & 0.001 \\
\hline $\begin{array}{l}\text { Predominant type of emphysema } \\
\text { (panlobular vs centrilobular) }\end{array}$ & 0.26 & $0.09-0.74$ & 0.018 \\
\hline Pleural adhesion & 0.33 & $0.13-0.83$ & 0.012 \\
\hline RV (change per unit) & 1.58 & $1.10-2.25$ & 0.012 \\
\hline $\begin{array}{l}\text { Volume of the untreated ipsilateral } \\
\text { lobe to the volume of the hemithor }\end{array}$ & 0.93 & $0.87-0.99$ & 0.017 \\
\hline 6-MWT (per 10 m change) & 1.05 & $1.00-1.10$ & 0.077 \\
\hline Target lobe LLL (vs RUL) & 0.28 & $0.06-1.33$ & 0.108 \\
\hline Target lobe LUL (vs RUL) & 0.95 & $0.19-4.74$ & 0.954 \\
\hline Target lobe RLL (vs RUL) & 0.23 & $0.03-1.83$ & 0.165 \\
\hline Fissure integrity & 1.16 & $0.99-1.36$ & 0.075 \\
\hline
\end{tabular}

Abbreviations: LAV, low attenuation volume; LLL, left lower lobe; LUL, left upper lobe; RLL, right lower lobe; RUL, right upper lobe; RV, residual volume; 6-MWT, 6-minute walk test. 


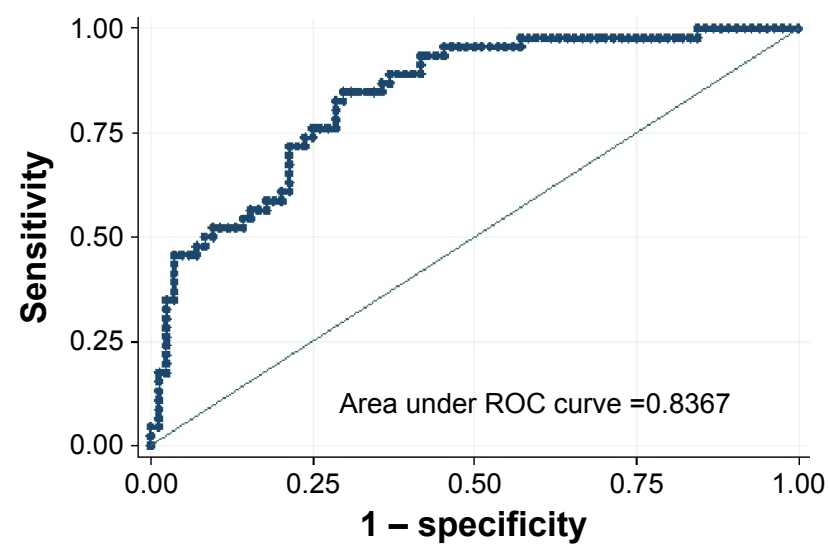

Figure 3 ROC curve.

Note: Prediction of pneumothorax following endoscopic valve therapy using eight CT and clinical parameters with an AUC of 0.8367 .

Abbreviations: $\mathrm{CT}$, computed tomography; AUC, area under the curve; ROC, receiver operating characteristic.

\section{Discussion}

Endoscopic valve therapy has evolved as an effective therapy for patients with severe COPD and emphysema. It has been developed as a minimally invasive technique to achieve lung volume reduction with less morbidity and mortality than has been reported in lung volume reduction surgery. ${ }^{15}$ However, valve therapy is also associated with potential complications. Over the past several years, the rate of pneumothorax following valve insertion particularly increased due to modified patient selection. A complete fissure is not only a predictor of excellent outcome but also of pneumothorax. Pneumothorax occurring in $18 \%-25 \%$ in patients undergoing valve insertion is actually the most common complication following valve therapy. ${ }^{4-7}$

To assess the risk of pneumothorax, we performed this retrospective analysis to determine the predictors of pneumothorax following valve insertion. As patients with TLVR and pneumothorax fulfill the criterion of complete interlobar fissure that is regarded as a prerequisite indication for valve therapy, we focused on both of these patient cohorts; the distinction between both these groups is crucial. As a result, the median of fissure integrity was $100 \%$, which explains why fissure integrity was not found to be a statistically significant predictor of pneumothorax.

In this analysis, the LAV\% of the untreated ipsilateral lobe, the volume of the untreated ipsilateral lobe to the volume of the hemithorax, the emphysema type, and pleural adhesions were significant CT predictors of pneumothorax. The higher the emphysematous destruction of the untreated ipsilateral lobe, the higher the risk of pneumothorax. The important role of the untreated ipsilateral lobe can be explained by the development of pneumothorax following valve implantation. It is hypothesized that pneumothorax occurs by parenchymal rupture in the adjacent untreated lung lobe in cases of rapid TLVR. In contrast to expectations, panlobular emphysema was found to be a protective factor for the development of pneumothorax. Initially it was assumed that panlobular emphysema distribution would increase the risk, but exactly the opposite was actually found. The blebs and bullae that were assumed to increase the risk were also found to play no significant role in the development of pneumothorax. The finding of the protective character of a panlobular emphysema can not be sufficiently explained; possibly, the compliance of the lung tissue in the different emphysema types varies and may be associated with a different risk of pneumothorax. In addition, pleural adhesions were surprisingly found to be protective factors against pneumothorax. As a clinical predictor, the residual volume was a variable that was independently associated with pneumothorax, adjusting for the target lobe. The higher the residual volume, the higher the risk of pneumothorax. A high residual volume may be associated with a greater volume shift, increasing the risk of parenchymal rupture. In that model, all of these parameters would predict whether a patient would experience a pneumothorax $84 \%$ of the time.

One retrospective trial demonstrated that most patients experienced an improvement in clinical outcomes due to the lung volume shift despite pneumothorax. ${ }^{8}$ This finding was confirmed by another trial demonstrating a statistically beneficial outcome in patients who developed pneumothorax following valve therapy. ${ }^{16}$ However, only patients in whom lobar atelectasis could be observed after recovering from pneumothorax will experience an outstanding improvement in lung function parameters, while patients without a significant TLVR following pneumothorax will experience slight but not relevant clinical improvement. In this analysis, no relevant clinical improvement was achieved after the development of a postinterventional pneumothorax, but pneumothorax following valve therapy did not impair the clinical outcome. Thus, there are some controversial data related to outcomes following pneumothorax in valve patients, but most studies suggest that patients who develop TLVR despite pneumothorax will benefit. However, pneumothorax is associated with prolonged hospitalization and immobilization, leading to muscle wasting and often requiring further intervention, such as chest tube insertion or surgical intervention. 
In one trial, bed rest and antitussive therapy successfully reduced the risk of pneumothorax following valve insertion. ${ }^{5}$ Forty patients who received modified medical care, including 48 hours of strict bed rest and $16 \mathrm{mg}$ codeine if needed for cough up to three times a day, were compared to 32 patients with standard medical care following valve therapy. While eight patients from the standard medical care group developed pneumothorax during hospitalization, only two from the modified medical care group experienced pneumothorax $(P=0.02)$. However, the number of treated patients in this study population was too low to allow a general statement for or against this modified postinterventional strategy.

Assessing the risk of pneumothorax helps to find the balance between efficacy and safety. Therefore, finding the predictors for pneumothorax is of great importance. The risk of pneumothorax of $18 \%-25 \%$ is accepted because the development of pneumothorax does not impair clinical patient outcomes. Nevertheless, the evaluation of valid prevention measures is crucial to minimize the risk of pneumothorax that presents a severe adverse event in patients with advanced emphysema.

In summary, pneumothorax is a commonly anticipated complication of valve therapy in patients with severe emphysema. Therefore, close monitoring following intervention is necessary. Patients with a high LAV\% of the untreated ipsilateral lobe and a high residual volume are at particularly great risk of pneumothorax. A high volume of the untreated ipsilateral lobe compared to volume of the hemithorax, panlobular emphysema and pleural adhesions, however, were found to be protective. A thorough evaluation of the risks is essential to determine and discuss the therapy and risk-benefit profiles with the patient.

\section{Acknowledgments}

The authors thank Dr Filippos Filippidis, Lecturer in Public Health at Imperial College, London, UK, for providing statistical advice.

\section{Author contributions}

All authors participated in trial coordination and study design and contributed toward data analysis, drafting and revising the paper, and agree to be accountable for all aspects of the work. The final submitted version was approved by all the authors.

\section{Disclosure}

DG: Lecture and travel fees from Pulmonx, Novartis, Astra Zeneca, Munidpharma, and Grifols. HL and VG: No conflicts of interest. RE: Lecture and travel fees from Pulmonx and
Olympus. FJFH: Consultant and lecture fee from Astra, Allmirall, Berlin Chemie, Boehringer, Roche, GSK, Pulmonx, PneumRx, Boston Scientific, Medupdate, Grifols, CSL Behring, Omniamed, Lilly, Novartis, Teva, Uptake, and Vital Air. CPH: Stock ownership in medical industry (Stada, GSK); Patents Method and Device for Representing the Microstructure of the Lungs. IPC8 Class: AA61B5055FI, PAN: 20080208038, Inventors: W Schreiber, U Wolf, AW Scholz, CP Heussel; Consultation or other fees (Schering-Plough 2009, 2010, Pfizer 2008-2014, Basilea 2008, 2009, 2010, Boehringer Ingelheim 2010-2014, Novartis 2010, 2012, Roche 2010, Astellas 2011, 2012, Gilead 2011-2014, MSD 2011-2013, Lilly 2011, Intermune 2013-2014, Fresenius 2013, 2014); Expert testimony (no); Research funding (Siemens 2012-2014, Pfizer 2012-2014, MeVis 2012, 2013, Boehringer Ingelheim 2015); Lecture fees (Gilead 2008-2014, Essex 2008, 2009, 2010, Schering-Plough 2008, 2009, 2010, AstraZeneca 2008-2012, Lilly 2008, 2009, 2012, Roche 2008, 2009, MSD 2009-2014, Pfizer 2010-2014, Bracco 2010, 2011, MEDA Pharma 2011, Intermune 2011-2014, Chiesi 2012, Siemens 2012, Covidien 2012, Pierre Fabre 2012, Boehringer Ingelheim 2012, 2013, 2014, Grifols 2012, Novartis 2013, 2014); Tobacco industry (no relationship). ME: Consultation fee Roche, 2015 and Novartis, 2015. The authors report no other conflicts of interest in this work.

\section{References}

1. Sciurba FC, Ernst A, Herth FJF, et al; VENT Study Research Group. A randomized study of endobronchial valves for advanced emphysema. N Engl J Med. 2010;363(13):1233-1244.

2. Herth FJ, Noppen M, Valipor A, et al; International VENT Study Group. Efficacy predictors of lung volume reduction with Zephyr valves in a European cohort. Eur Respir J. 2012;39(6):1334-1342.

3. Davey C, Zoumot Z, Jordan S, et al. Bronchoscopic lung volume reduction with endobronchial valves for patients with heterogeneous emphysema and intact interlobar fissures (the BeLieVeR-HIFI study): a randomised controlled trial. Lancet. 2015;386(9998):1066-1073.

4. Klooster K, ten Hacken NH, Hartman JE, Kerstjens HA, van Rikxoort EM, Slebos DJ. Endobronchial valves for emphysema without interlobar collateral ventilation. $N$ Engl J Med. 2015;373(24):2325-2335.

5. Herzog D, Poellinger A, Doellinger F, et al. Modifying post-operative medical care after EBV implant may reduce pneumothorax incidence. PLoS One. 2015;10(5):e0128097.

6. Gompelmann D, Eberhardt R, Herth F. Endoscopic volume reduction in COPD: a critical review. Dtsch Arztebl Int. 2014;111(49):827-833.

7. Lepper PM, Ballek D, Trudzinski F, et al. Endoscopic lung volume reduction using endobronchial valves in patients with very low FEV1. Am J Respir Crit Care Med. 2014;189:A3052.

8. Gompelmann D, Herth FJF, Slebos DJ, et al. Pneumothorax following endobronchial valve therapy and its impact on clinical outcomes in severe emphysema. Respiration. 2014;87(6):485-491.

9. Valipour A, Slebos DJ, de Oliveira HG, et al. Expert statement: pneumothorax associated with endoscopic valve therapy associated with endoscopic valve therapy for emphysema - potential mechanisms, treatment algorithm, and case examples. Respiration. 2014;87(6):513-521.

10. Christ M, Dormann H, Enk R, et al. Chest pain units or chest pain algorithm? Med Klin Intensivmed Notfmed. 2014;109(7):495-503. 
11. Koyama H, Ohno Y, Yamazaki Y, et al. Quantitative and qualitative assessments of lung destruction and pulmonary functional loss from reduced-dose thin-section CT in pulmonary emphysema patients. Acad Radiol. 2010;17(2):163-168.

12. Tanabe N, Muro S, Tanaka S, et al. Emphysema distribution and annual changes in pulmonary function in male patients with chronic obstructive pulmonary disease. Respir Res. 2012;13:31.

13. Park KJ, Bergin CJ, Clausen JL. Quantitation of emphysema with three-dimensional CT densitometry: comparison with two-dimensional analysis, visual emphysema scores, and pulmonary function test results. Radiology. 1999;211(2):541-547.
14. Suzuki T, Tada Y, Kawata N, et al. Influence of pulmonary emphysema on COPD assessment test-oriented categorization in GOLD document. Int J Chron Obstruct Pulmon Dis. 2015;10:1199-1205.

15. Fishman A, Martinez F, Naunheim K, et al. A randomized trial comparing lung-volume-reduction surgery with medical therapy for severe emphysema. N Engl J Med. 2003;348(21):2059-2073.

16. Gompelmann D, Herth FJF, Ehlken N, Heussel CP, Hoffmann H, Eberhardt R. Development of endoscopic valve therapy in patients with severe emphysema. ERS. 2016;46(Suppl 59):PA795.

International Journal of COPD

\section{Publish your work in this journal}

The International Journal of COPD is an international, peer-reviewed journal of therapeutics and pharmacology focusing on concise rapid reporting of clinical studies and reviews in COPD. Special focus is given to the pathophysiological processes underlying the disease, intervention programs, patient focused education, and self management protocols.

\section{Dovepress}

This journal is indexed on PubMed Central, MedLine and CAS. The manuscript management system is completely online and includes a very quick and fair peer-review system, which is all easy to use. Visit http://www.dovepress.com/testimonials.php to read real quotes from published authors.

Submit your manuscript here: http://www.dovepress.com/international-journal-of-chronic-obstructive-pulmonary-disease-journal 